\title{
Entrelacs
}

ENTRELACS Cinéma et audiovisuel

18 | 2021

Laurent Roth, cinéaste

\section{Une archive au présent. Le principe de renaissance}

Julie Savelli

\section{OpenEdition}

Journals

Édition électronique

URL : https://journals.openedition.org/entrelacs/6188

DOI : 10.4000/entrelacs. 6188

ISSN : 2261-5482

Éditeur

Éditions Téraèdre

Référence électronique

Julie Savelli, «Une archive au présent. Le principe de renaissance », Entrelacs [En ligne], 18| 2021, mis en ligne le 13 décembre 2021, consulté le 15 décembre 2021. URL : http://journals.openedition.org/ entrelacs/6188; DOI : https://doi.org/10.4000/entrelacs.6188

Ce document a été généré automatiquement le 15 décembre 2021.

Tous droits réservés 


\title{
Une archive au présent. Le principe de renaissance ${ }^{1}$
}

\author{
Julie Savelli
}

\section{Remerciements : Laurent Roth et Jacques Gerstenkorn.}

1 L'Emmuré de Paris est un photo-film dans la droite lignée du photo-roman La Jetée. Dans les deux récits, la continuité s'élabore à partir d'images fixes pour donner l'illusion du mouvement tandis que la photographie se fait le réservoir d'un temps subjectif, lacunaire et ouvert : une « archive au présent », selon le réalisateur Laurent Roth.

Film fait seulement de voix, de sons et de photos, c'est un nouveau défi pour moi !

La photographie n'est pas ici un handicap, une forme de "cinéma diminué ». Sa

fixité, bien au contraire, me semble révolutionnaire : image figée, elle est déjà une " archive », mais une archive au présent, qui me permet de documenter, indiquer, accuser, dénoncer ce danger de la pétrification qui menace Paris².

Dans son livre Mal d'archive ${ }^{3}$, Jacques Derrida rappelle cette capacité qu'a l'archive de nous mettre en présence de quelque chose dont elle est le prolongement et qu'elle recommence - une capacité inscrite dans son concept même, ou plutôt dans l'étymologie grecque arkhé sur laquelle est construit le terme d'archive - qui signifie à la fois le principe et le commencement d'un être. Ainsi, selon cette interprétation généalogique de l'archive, chaque geste photographique dans L'Emmuré de Paris se trouve vivement amplifié par l'éclat que lui procure son statut de trace ou de reste. L'image fixe nous met au contact de la réalité dont elle résulte, laquelle de ce fait resurgit sous une forme nouvelle dans l'intervalle qui sépare la prise de vue photographique de sa genèse. Ce principe de renaissance à et en soi caractérise l'image-archive mais plus largement le projet de L'Emmuré de Paris dans son ensemble.

3 La gestation de ce dernier-né comprend déjà en elle-même un nombre infini de renaissances. Si ce nouveau film autobiographique touche aujourd'hui à sa fin, il faut rappeler que son projet a débuté il y a plus de dix ans, en 2010, dans le cadre d'une résidence d'artistes au studio Centquatre à la Villette. Ce work in progress a connu de nombreux rebondissements génétiques durant sa production, au fur et à mesure que 
s'étoffait la recherche menée par le cinéaste sur l'histoire et l'architecture du quartier mais aussi sur son actualité ordinaire.

Le pari a tout de suite été de produire ce film sur la durée. La légèreté de l'équipe a permis de couvrir des événements imprévus (l'installation de sans-papiers passage de Crimée), de tenir le journal des chantiers (la destruction et reconstruction de la Tour Maroc, la démolition des pavillons des Halles et la construction de la canopée actuellement visible, les travaux de restauration du Panthéon), de se fondre dans la foule (cérémonies des Morts de la Rue et flash-mobs sur la place Stalingrad).

4 Le film a ainsi évolué et mis du temps à trouver sa forme alternant périodes de montage, nouveaux shootings et projections tests en festivals, écoles d'art, musées. L'Emmuré de Paris s'est donc réalisé dans le temps long au gré des rencontres avec les publics ${ }^{4}$. Les premières versions longues ont été intitulées Arc, Arceaux, Arcades avant de se recentrer sur le personnage (interprété par le cinéaste) et son enfermement, devenant L'Emmuré de Paris. Les différentes tenues vestimentaires (veste beige puis caban d'hiver bleu) ainsi que les traces d'un léger vieillissement du protagoniste à l'écran, sont des marqueurs qui traduisent l'épaisseur temporelle du projet. Si la durée de la réalisation a sans aucun doute été éprouvante pour l'auteur ${ }^{5}$, elle a aussi permis à Laurent Roth de perfectionner l'ensemble, notamment en lui donnant une concrétisation technique optimale. Il a par exemple, encore très récemment, expérimenté et intégré de nouveaux effets spéciaux, assumant pleinement de jouer avec les codes de genre (la BD, la SF). Par ailleurs, Laurent Roth n'a cessé de reprendre et d'enrichir son récit notamment du point de vue sonore avec de nouvelles compositions musicales, le réenregistrement des voix du commentaire en studio ou l'ajout de bruitages. Si L'Emmuré de Paris continue de renaître toujours et encore sous des formes grandies, repoussant en quelque sorte son achèvement, il est actuellement en postproduction et s'achemine donc vers sa finalisation. L'endurance de cette gestation «monstrueuse » doit être comprise comme un facteur de stimulation et de renouvellement permanent qui structure intimement la mise en œuvre.

5 Ce principe génétique de renaissance se traduit aussi dans la revisitation décomplexée de La Jetée dont l'ombre plane indéniablement sur L'Emmuré de Paris. La nouvelle " fantaisie documentaire ${ }^{6}$ » de Laurent Roth se présente en effet comme un voyage dans le temps, associant l'histoire de la ville de Paris à l'actualité la plus récente - l'évocation des attentats terroristes de 2015 ou du mouvement des « gilets jaunes » par exemple. Ce récit uchronique filmé au banc-titre témoigne en outre d'une poétique markerienne du détail et du recadrage (on retrouve aussi les rôles muets et la puissance narrative du commentaire). En revanche Laurent Roth marque un double écart vis à vis du maître en faisant le choix de l'absurde et de l'animation. Il entreprend une enquête culturelle et historique propice au fantasme généalogique, endossant les traits de son personnage tragique - le Fou, l'Idiot - que l'on avait vu apparaître pour la première fois dans le court métrage Une maison de famille $(2004)^{7}$ puis dans sa version longue J'ai quitté l'Aquitaine (2005).

6 Ce double asilaire du héros markerien, Davos Hanich, est placé dans un périmètre volontairement restreint à un pâté de maisons du quartier de la Villette (rue d'Aubervilliers, rue de Crimée, avenue de Flandre, boulevard de la Chapelle). Dans ce confinement topographique, l'Emmuré est assigné au recensement méthodique des arcs, arceaux et arcades. Cette thérapie délirante - instituée par la figure alter ego du médecin-chef interprétée par la complice Kendra Walker - constitue un arc narratif prétexte à arpenter une des banlieues populaires de Paris «qui n'intéresse aucun 
touriste et pourtant vrai laboratoire de ce que pourrait être l'intégration des banlieues à la métropole ". Car ce récit partage bien la filiation critique de La Jetée mais dans un registre loufoque qui recourt aux technologies de l'image. Ainsi la mixité sociale visible dans les photographies du présent cohabite avec l'histoire de la ville de Paris qui se matérialise et renaît sous nos yeux en empruntant aux effets spéciaux leur puissance d'incarnation. Laurent Roth cherche à lutter contre la fabrique de l'oubli et à renouer avec une mémoire enfouie: il pointe dans un même mouvement la disparition du peuple relégué extramuros, aux portes de Paris, et la liquidation de l'histoire affiliée à la cartographie de ce quartier périphérique, une histoire marginale qui demeure ignorée.

Film-fleuve, L'Emmuré de Paris charrie tout un imaginaire qui associe l'ordinarité du contemporain aux fantômes des grands hommes de la Villette. Le brouillage des temporalités est ici une manière d'échapper à l'enfermement réel et symbolique occasionné par les troubles de la personnalité dont souffre le personnage de Laurent Roth en même temps que de renaître à soi-même.

La prétendue aliénation mentale de mon personnage lui permet [...] de chevaucher les espaces et les époques librement, d'incarner plusieurs personnages à la fois, et surtout de faire rire (je l'espère), tout en délivrant des contenus savants, politiques et poétiques. C'est un griot qui raconte une autre histoire de France, tangentielle et minoritaire.

Puisque "Je est un autre ${ }^{8}$ ", selon la célèbre formule d'Arthur Rimbaud, le cinéaste s'applique à devenir cet autre moi qui n'est pas moi. L'Emmuré de Paris est pensé sur le mode de l'enchâssement des figures qui, telles des poupées russes, s'imbriquent curieusement l'une dans l'autre. Ainsi, le personnage de l'Emmuré traverse le quartier de la Villette et comme ces transformistes dans les foires de jadis, se métamorphose en présence des lieux empreints de mémoire. Le corps du cinéaste a dès lors une fonction narrative et imaginale, accueillant des choré-singularités multiples, celles des agents de l'histoire de Paris qui possèdent successivement l'Emmuré. La transe est brève et opère par la voix qui accompagne le morphing du visage à l'image. Parmi ces génies, « ces grands hommes ", il y a Auguste Blanqui (1805-1881), républicain socialiste visionnaire surnommé «L'Enfermé », qui tente d'allumer un foyer insurrectionnel à la Villette et échoue à mettre à bas le second Empire - il est le chef dit-on qui aurait cruellement manqué à la Commune de Paris.

Oui, Messieurs, c'est la guerre entre les riches et les pauvres : les riches l'ont voulu ainsi ; ils sont en effet les agresseurs. Seulement ils considèrent comme une action néfaste le fait que les pauvres opposent une résistance. Ils diraient volontiers, en parlant du peuple : cet animal est si féroce qu'il se défend quand il est attaqué9.

9 Il y a aussi Jacob Rodrigue Pereire (1715-1780), instituteur juif précurseur de l'éducation des sourds-muets et de l'orthophonie - dont les travaux engagés sont aujourd'hui méconnus - qui achète un terrain rue de Flandre pour y instituer un cimetière pour les juifs portugais de la capitale.

Je suis mort depuis longtemps. J'ai construit ce cimetière. À peine achevé, j'y ai enterré mon fils premier né puis je l'ai suivi le 4 septembre 1780. Je m'appelle Jacob Rodrigue Pereire ${ }^{10}$.

Ou encore le Maréchal Marmont (1774-1852), héros maudit de la bataille de Paris, qui signe la capitulation de l'Empire à la barrière des Vertus (Stalingrad) en mars 1814 - il sauve Paris de la destruction, mais passe du mauvais côté, se ralliant à Louis XVIII.

11 Restés en marge de l'histoire, ignorés en raison de leur échec, de leur religion ou de leur traitrise, force est de constater que ces fantômes de la Villette sont tous frappés 
par un destin tragique. La "fatalité de la relégation », selon les termes de Laurent Roth, semble être la marque du quartier lui-même. Faubourg Nord-Est de la capitale, la Villette est à la fois un foyer du Paris révolutionnaire au XIX ${ }^{e}$ siècle et le lieu de passage des armées et des invasions :

[...] le vide-ordure de Paris, avec son gigantesque dépotoir pour les vidangeurs, ses «voiries» (décharges à ciel ouvert), ses pompes funèbres, ses abattoirs, ses industries polluantes, à commencer par les énormes cuves du gazomètre, ses champs d'équarrissage, ses rats et ses corbeaux charognards... L'esprit du Sang des bêtes ${ }^{11}$ est encore là qui plane sur le quartier, malgré le remodelage du périmètre durant ces cinquante dernières années.

12 Si hier comme aujourd'hui, la Villette pâtit de son caractère populaire, les ancêtres convoqués par le personnage de l'Emmuré exposent sous un nouvel angle « la nature héroïque » d'un quartier peu considéré par l'historiographie académique. Ces microrécits généalogiques opèrent des percées temporelles qui mettent immédiatement en perspective le présent: pas de démonstration ou de grands discours mais les visages multicolores des passants circulant dans le cadre, et aussi quelques rencontres singulières - avec Amadou par exemple : « Nous promettons de nous revoir ${ }^{12}$ ». Laurent Roth incarne merveilleusement ici le précepte de Walter Benjamin selon qui une histoire ne vaut que si elle donne voix aux anonymes. À cette lignée des grands hommes, il faudrait donc ajouter la figure benjaminienne de l'historien-chiffonier ${ }^{13} \mathrm{car}$ l'Emmuré - déterminé à se saisir de toute trace d'histoire pour nous rendre sensibles à leur infamie ${ }^{14}$ - fait dit-il « du lieu du rebut le lieu du combat».

Souffrant de confusion mentale, le personnage de Laurent Roth participe ici d'un jeu de masques qui paradoxalement permet de déplacer le regard, de prêcher le faux pour accéder à travers chaque délire généalogique à une vérité personnelle. Après en avoir découvert une première (je suis ce révolutionnaire), il en découvre une seconde (je suis ce juif éclairé), puis une autre (je suis ce héros maudit), et encore d'autres (" Je suis un étudiant en fuite, un immigré usé, une prostituée finissante, un soldat éclopé » dit l'Emmuré). La dernière vérité est celle de l'avenir : «Je suis cet enfant ». La sentence est énoncée à deux reprises dans le film avant d'être immédiatement récusée - «Je ne suis pas cet enfant ». Il faut attendre la troisième occurrence, à la fin du récit, pour que l'affirmatif l'emporte, actualisant par le verbe la guérison du patient, sa renaissance ou peut-être même sa rédemption (si l'on se fie à la vieille nonne qui apparaît miraculeusement quelques plans plus tôt ${ }^{15}$ ). Il ne s'agit pas ici d'échapper à soi mais bien de renaître en l'autre pour mieux se connaître. À sa manière fantaisiste, Laurent Roth réactive dans L'Emmuré de Paris le principe des altérités incluses ${ }^{16}$ qui est à l'œuvre dans des civilisations fort anciennes, ou lointaines. Il expérimente cette nécessité archaïque de recourir à l'autre dans la réalisation de soi : devenir l'autre en soi (ou réciproquement) permet dès lors d'élargir et de reconfigurer sa généalogie au prisme du commun. 


\section{NOTES}

1. Ce texte est tiré d'une publication parue dans la revue À l'épreuve qui porte sur l'inspiration markerienne commune aux projets en cours de Dominique Cabrera et de Laurent Roth («L'autresoi à l'écran. À propos du commun généalogique et de l'ombre bienveillante de l'animal Marker ", $\left.\mathrm{n}^{\circ} 7,2021\right)$.

2. Toutes les citations de Laurent Roth à propos de son film L'Emmuré de Paris sont reproduites avec son accord et extraites d'un dossier de présentation présenté au CNAP (Centre National des Arts Plastiques) le 16 mars 2016.

3. Jacques Derrida, Mal d'archive : une impression freudienne, Paris, Galilée, coll. « Incises », 1995.

4. Le film a d'abord été présenté dans une version « fin de résidence » de 15 minutes avec un commentaire improvisé au micro par son auteur lors de la Nuit blanche en 2010, puis à des étapes de fabrication plus avancées dans le cadre des Rencontres cinématographiques de Pézenas en février 2012 ou à Visions du Réel à Nyon en avril 2012. Ces informations sur la genèse du film proviennent d'un entretien avec Laurent Roth réalisé en collaboration avec Jacques Gerstenkorn en septembre 2020 en vue de la publication de ce numéro spécial consacré aux films du cinéaste. Voir : « Dans la fabrique de L'Emmuré de Paris. Genèse d'une fantaisie documentaire ».

5. À mi-parcours, Laurent Roth a dû faire face à la cessation d'activité de la société de production Inthemood, porteuse du projet.

6. Le terme est utilisé au générique pour présenter le film: "L'Emmuré de Paris. Une fantaisie documentaire de Laurent Roth».

7. Voir dans ce volume: Rémi Fontanel, «Une maison de famille : une expérience cinématographique de l'intime ».

8. Arthur Rimbaud à Paul Demeny (Lettre du Voyant, 15 mai 1871).

9. Extrait de la défense d'Auguste Blanqui en Cour d'Assises, en 1832, cité dans le commentaire du film (version de travail novembre 2020 - 12'07").

10. Commentaire de L'Emmuré de Paris (version de travail novembre 2020 - 23'42").

11. Le Sang des bêtes (Georges Franju, 1949) est filmé dans les abattoirs parisiens de la Villette et de Vaugirard.

12. Commentaire de L'Emmuré de Paris (version de travail novembre 2020 - 16'18").

13. «Méthode de travail : montage littéraire. Je n'ai rien à dire. Seulement à montrer. Je ne vais rien dérober de précieux, ni m’approprier aucune formule spirituelle. Les haillons, les déchets, eux, je ne veux pas les inventorier, mais leur rendre justice de la seule façon possible: les utiliser. » (Walter Benjamin, Paris, capitale du XIXe siècle, 1935, dans Euvres, Paris, Gallimard, 2000, p. 60.)

14. Michel Foucault, « La vie des hommes infâmes » [1977], dans Archives de l'infamie, Paris, Les Prairies ordinaires, 2009.

15. Laurent Roth est d'origine juive et de confession catholique. Il joue de ce palimpseste dans la séquence "du cimetière juif» en incrustant une goutte de sang sur la paume de sa main, devenant dès lors une icône du Christ.

16. La notion d' " altérité incluse » a été proposée par le collectif de chercheurs du centre Louis Gernet (historiens de Rome et de la Grèce ancienne) de l'EHESS: «c'est un phénomène d'appropriation de l'autre en conservant ou en exaspérant son altérité afin de construire sa propre identité ». Florence Dupont et Emmanuelle Valette-Cagnac (dir.), Façons de parler grec à Rome, Paris, Belin, «L'Antiquité au présent », 2005, p. 257. Voir aussi : Claire Cécile Mitatre, « Eston toujours le "barbare" des autres?", dans Maxime Del Fiol et Claire Cécile Mitatre, Les Occidents des mondes arabes et musulmans. Afrique du Nord - XIX ${ }^{e}-X X I^{e}$ siècles, Paris, Éditions Geuthner, 2018. 


\section{RÉSUMÉS}

Cette contribution explore la conduite créatrice qui préside à la mise en œuvre d'un « autre-soi » dans L'Emmuré de Paris, premier long métrage du cinéaste Laurent Roth. Non seulement cette fantaisie documentaire à la première personne fait émerger les nœuds de la subjectivité et de l'altérité mais elle convoque aussi un héritage sensible où les proches, les anonymes et les grands hommes sont pris, sur un principe d'inclusion, dans de nouvelles relations de parenté. Il apparaît que l'imaginaire identitaire produit ici des frottements avec la fiction et l'expérimentation éclairant la part de l'autre dans la construction de soi et par là-même notre sensibilité contemporaine.

This contribution explores the creative process that presides over the implementation of an "other-self" in L'Emmuré de Paris, the first feature film by filmmaker Laurent Roth. Not only does this first-person documentary fantasy bring out the knots of subjectivity and otherness, but it also summons a sensitive heritage in which relatives, anonymous people and great men are caught up, on a principle of inclusion, in new relationships of kinship. It appears that the imaginary identity here produces friction with fiction and experimentation, shedding light on the part of the other in the construction of the self and thus on our contemporary sensitivity.

\section{AUTEUR}

\section{JULIE SAVELLI}

Maîtresse de conférences en études cinématographiques et audiovisuelles à l'université PaulValéry Montpellier 3 et membre du Centre de recherches RiRRa21. Dans ses travaux qui portent sur la création documentaire contemporaine, elle s'intéresse plus particulièrement au cinéma engagé, à l'autobiographie en images et à l'anthropologie du visuel. Parmi ses diverses publications, mentionnons la direction de trois ouvrages collectifs en 2021 : Jean Rouch. Passeur d'images, passeur de mondes (Téraèdre), John Cassavetes. Imaginaires des corps, entre la scène et l'écran (PUP) et Dominique Cabrera. L'intime et le Politique (De l'Incidence Éditeur). 